\title{
Revisão integrativa: intercambialidade e aspectos farmacoeconômicos dos medicamentos biossimilares
}

\author{
Emanuela Pires SILVA ${ }^{(1)}$ \\ Lúcia Dias da Silva GUERRA ${ }^{(1)}$ \\ ${ }^{(1)}$ Faculdade de Saúde Pública, Universidade de São Paulo - USP, São Paulo, SP, Brasil.
}

Recebido: 28 jan 2019 Aceito: 10 fev 2019

Autor de correspondência: emanuela.nela@gmail.com

Conflito de interesses: Os autores declaram não haver nenhum interesse profissional ou pessoal que possa gerar conflito de interesses em relação a este manuscrito.

\section{Resumo}

Os medicamentos biológicos provêm de organismos vivos, que foram modificados com recurso da biotecnologia. Essa modificação faz com que os organismos ou células vivas produzem a substância ativa do medicamento biológico. Estas substâncias ativas (por exemplo, proteínas) são normalmente maiores e mais complexas do que as dos medicamentos não biológicos. Entre os medicamentos biológicos disponíveis estão os hormônios como a insulina e de crescimento, bem como anticorpos monoclonais utilizados no tratamento de doenças autoimunes e das neoplasias. O medicamento biossimilar é desenvolvido de modo a ser altamente similar a um medicamento biológico existente. Este medicamento biológico existente é um medicamento que já foi aprovado e registrado pela Agência de Vigilância Sanitária - Anvisa, e é designado por medicamento de referência. Quando este deixa de estar protegido pela respectiva patente e o seu período de exclusividade termina, o medicamento biossimilar pode ser introduzido no mercado. Por ser altamente similar, entendese que o medicamento biossimilar e o seu medicamento de referência são essencialmente iguais, embora possam existir ligeiras diferenças nas suas substâncias ativas. Tais diferenças decorrem do facto de estas substâncias ativas serem normalmente moléculas grandes e complexas e serem produzidas por células vivas. Quaisquer diferenças entre o medicamento biossimilar e o seu medicamento de referência são mantidas dentro de limites estreitos para assegurar que ambos funcionam da mesma forma. Há questões importantes sobre o tema, como a extrapolação de indicações, isto é, a aprovação de um biossimilar para uso em todas as indicações do medicamento de referência. Já a intercambialidade consiste na troca de um medicamento por outro produto com eficácia e modo de ação comprovadamente equivalentes sem o risco de um desfecho de saúde adverso, trata-se de um pré-requisito para a substituição. $A$ substituição é caracterizada pela troca de um medicamento inovador pelo biossimilar, ou um biossimilar por outro, a nível de farmácia, sem consentimento prévio do médico prescritor. Os altos preços e o sucesso dos medicamentos de referência pressionam os custos em serviços de saúde, o que pode levar a uma barreira para acesso de usuários a produtos biológicos. Com o custo crescente de serviços de saúde e a pressão econômica para reduzir e/ou sustentar o gasto com 
esses serviços, os biossimilares podem ser determinantes para reduzir o custo de medicamentos biológicos e garantindo a manutenção e ampliação de acesso de usuários ao tratamento. Uma vez que os medicamentos biossimilares tendem a ser mais acessíveis aos usuários e aos sistemas de saúde devido ao menor investimento de recursos em pesquisa e desenvolvimento ( $P \& D)$ e do possível impacto da concorrência de mercado. Tendo em vista a necessidade de orientar e coordenar ações específicas sobre a utilização de medicamentos biológicos no âmbito do SUS, o Ministério da Saúde instituiu o grupo de trabalho (GT) da Política Nacional de Medicamentos Biológicos no âmbito do Sistema Único de Saúde - SUS, por meio da Portaria GM/MS n 1.160 de 05/2018, que tem como finalidade discutir os temas pertinentes à pesquisa, desenvolvimento, produção, regulação, acesso e monitoramento do uso de medicamentos biológicos fornecidos pelo SUS. Essa medida se deve ao alto custo ser consistentemente apresentado como uma das principais barreiras de acesso a medicamentos biológicos no SUS, espera-se que a redução de preços por meio de competição seja observada com a entrada de biossimilares no mercado e que possibilite um maior acesso ao tratamento. Para tanto, este estudo tem o objetivo de analisar a intercambialidade de medicamentos biossimilares e suas possíveis vantagens econômicas em comparação aos medicamentos biológicos de referência. Objetivo: analisar a intercambialidade de medicamentos biossimilares e suas possíveis vantagens econômicas em comparação aos medicamentos biológicos de referência. Método: neste estudo optou-se por uma metodologia de revisão sistemática integrativa da literatura, que tem por definição reunir e sintetizar sistematicamente o conhecimento científico já produzido sobre uma determinada temática, possibilitando uma compreensão abrangente do problema em questão (Whittemore, 2005). Para a identificação dos descritores de busca sobre o tema proposto foi utilizada o Descritores em Ciências da Saúde - DeCS. A seleção dos descritores a serem empregados na busca foi feita considerando a variedade de termos empregados como sinônimos no contexto brasileiro e os principais descritores de busca foram: medicamentos biossimilares, biotecnologia, medicamentos biológicos, farmacoeconomia, economia da saúde, gastos em saúde e intercambialidade de medicamentos. Então, foi realizada uma busca ativa de informações nas bases de dados Literatura Latino-Americana e do Caribe em Ciências da Saúde - LILACS e devido ao baixo número de artigos relacionados ao tema, optou-se pela busca da literatura na base de dados PubMed (Base da dados científica desenvolvida pela National Center for Biotechnology Information - NCBI da National Library of Medicine - NLM) utilizando a ferramenta MeSH (Medical Subject Headings) para verificação dos descritores. Os descritores encontrados no DeCs e PubMed foram medicamentos biossimilares; biotecnologia; medicamentos biológicos; intercambialidade de medicamentos; farmacoeconomia; economia da saúde; e gastos em saúde; e em inglês biosimilar pharmaceuticals, biotechnology, follow on biologics, drug switching, therapeutic substitution; drug substitution; pharmacoeconomics, drug costs, e technology assessment, health. Para a elaboração da sintaxe, os descritores foram 
distribuídos em três partes - População ("biosimilar pharmaceuticals", "biotechnology", "follow on biologics"), Fenômeno ("drug switching", " therapeutic substitution", "drug substitution") e Contexto ("pharmacoeconomics", "drug costs" e "technology assessment, health"). Considerações finais: a partir deste modelo a busca no PubMed resultou em 55 estudos, com a seguinte sintaxe: (((Biosimilar Pharmaceuticals OR Biotechnology OR Follow on Biologics)) AND (Drug Switching OR Therapeutic Substitution OR Drug Substitution)) AND (Pharmacoeconomics OR Drug Costs OR Technology Assessment, Health); Notase, a partir de uma análise preliminar que todos os artigos são estrangeiros e os artigos que abordam os tópicos biossimilares e economia surgem a partir de 2013.

Descritores: Medicamentos Biossimilares; Substituição de Medicamentos; Farmacoeconomia; Medicamentos Biossimilares. 